\title{
An evaluation of GPUs for use in an upgraded ATLAS High Level Trigger
}

\author{
A. T. Delgado, P. Conde Muíño, J. Augusto Soares, R. Gonçalo, J. Baines, T. Bold, D. Emeliyanov, S. Kama, M. \\ Bauce, A. Messina, M. Negrini, A. Sidoti, L. Rinaldi, S. Tupputi, Z. D. Greenwood, A. Elliott, S. Laosooksathit \\ On behalf of the ATLAS Collaboration
}

\begin{abstract}
1 Abstract-ATLAS is a general purpose particle physics experi- 322 the LHC is operating at a centre-of-mass energy of $13 \mathrm{TeV}$ 2 ment located on the LHC collider at CERN. The ATLAS Trigger ${ }_{33}$ to $14 \mathrm{TeV}$, almost two times higher than in Run 1 , and an ${ }_{3}$ system consists of two levels, the first level (L1) implemented in ${ }_{34}$ average of $\sim 27$ pp collisions per bunch crossing (known as 4 hardware and the High Level Trigger (HLT) implemented in ${ }_{5}^{34}$ aftware running on a computing cluster of commodity CPUs.

${ }_{6}$ The HLT reduces the trigger rate from the $100 \mathrm{kHz} \mathrm{L1}$ accept ${ }^{36}$ The third data taking period, known as Run 3, is scheduled 7 rate to $1 \mathrm{kHz}$ for recording, requiring an average per-event ${ }_{37}$ to start in 2019 , after a two years shutdown for upgrade of 8 processing time of $\sim \mathbf{3 0 0} \mathbf{~ m s}$ for this task. The HLT selection is ${ }_{38}$ the accelerator and the detectors, as shown on the activity ${ }_{9}$ based on reconstructing tracks in the Inner Detector and Muon ${ }_{39}$ schedule Table I. In Run 3 the LHC will work with a two times ${ }_{10}$ Spectrometer and clusters of energy deposited in the calorimeters 11 (electromagnetic and hadronic). Performing this reconstruction 12 within the available HLT computing cluster resources presents a 4 ${ }_{13}$ significant challenge. Future HLT upgrades will result in higher ${ }_{42}$ ber of particles hitting the detectors per event. As this results ${ }_{14}$ detector occupancies and, consequently, will harden the recon- ${ }_{43}$ in events that are more complex, the trigger reconstruction 15 struction constraints. General purpose Graphics Processor Units 44 software will demand more computing power. Therefore it will ${ }_{16}$ (GPGPU) are being evaluated for possible future inclusion in an ${ }_{45}$ be essential to reduce the processing time of the reconstruction 17 upgraded HLT computing cluster. We report on a demonstrator ${ }^{45}$ be that has been developed consisting of GPGPU implementations of 46 algorithms, to keep them within the time constraints of the
18 , 19 the calorimeters clustering and Inner Detector and Muon track- 47 online system while maintain the same physics performance. 20 ing algorithms integrated within the HLT software framework. ${ }_{48}$ The General Purpose Graphical Processing Units (GPGPUs) ${ }_{21}$ We give a brief overview of the algorithm implementation and ${ }_{49}$ can provide better computing performance to power ratio than ${ }_{22}$ present preliminary measurements comparing the performance ${ }_{50}^{49}$ Central Processing Units (CPUs), and are thus good candidates ${ }_{23}$ of the GPGPU algorithms with the current CPU versions. ${ }_{51}$ to maximize the computing cluster power, as the computing ${ }_{52}$ cluster is limited by the rack-space and cooling power.
\end{abstract}

\section{INTRODUCTION}

${ }^{x} \mathbf{T}$ HE CERN Large Hadron Collider (LHC) [1] was build ${ }_{53} A$. The ATLAS experiment

to explore the fundamental constituents of nature and the ${ }_{54}$ The ATLAS detector is one of the two LHC multi purpose ${ }_{27}$ forces between them, at unprecedented energies. It is a circular ${ }_{55}$ experiments [4].

28 accelerator with a perimeter of $27 \mathrm{~km}$ where two proton beams ${ }_{56}$ It is a cylindrical shape detector with $46 \mathrm{~m}$ length and ${ }_{29}$ cross 40 million times per second. Each beam crossing is ${ }_{57} 25 \mathrm{~m}$ height. The detection elements are arranged in layers 30 usually referred to as an event. The second data taking period, ${ }_{58}$ around the beam pipe. The inner part is the Inner Detector ${ }_{31}$ Run 2, started this year and will last until 2018. During Run ${ }_{59}$ tracker (ID), immersed in a magnetic field generated by

Manuscript received November 23, 2015. A. T. Delgado acknowledges ${ }_{60}$ a superconductor solenoid. The ID allows the detection of the support of the IDPASC doctorate network and Fundação para a Ciência e Tecnologia, Portugal, through the grant SFRH/BD/51792/2011 and the FEDER/COMPETE-QREN.

A. T. Delgado, P. Conde Muíño, J. Augusto Soares and R. Gonçalo are with the Laboratório de Instrumentação e Física Experimental de Partículas (LIP), Lisbon, Portugal

J. Augusto Soares is also with INESC-ID and Faculdade de Ciências, Universidade de Lisboa, Lisboa, Portugal

J. Baines and D. Emeliyanov are with STFC and Rutherford Appleton Lab, GB

T. Bold is with AGH Univ. of Science and Technology, Krakow, Poland S. Kama is with Southern Methodist University, Dallas, TX, USA

M. Bauce and A. Messina are with Sapienza Università di Roma and INFN, Italy

M. Negrini and A. Sidoti are with Istituto Nazionale di Fisica (INFN), Italy

L. Rinaldi is with Università di Bologna and INFN, Italy

S. Tupputi is with INFN-CNAF, Italy

Z. D. Greenwood, A. Elliott and S. Laosooksathit are with Louisiana Tech

University, Ruston LA, USA

(e-mail: tavares@cern.ch).

${ }_{61}$ charged particles trajectories and is made of three different 62 technologies: pixel detectors, in the inner most layers; Semi${ }_{63}$ conductor Tracker (SCT) on the middle layers and Transition ${ }_{64}$ Radiation Tracker (TRT) in the outer most layers.

${ }_{65}$ The ID is surrounded by the calorimeter systems, composed 66 by the electromagnetic calorimeter, based on Liquid Argon ${ }_{67}$ (LAr) technology, and the hadronic calorimeters, made of LAr 68 and scintillator tile technologies.

69 The muon spectrometer is the outermost sub-detector, im70 mersed in a second magnetic field generated by superconduct71 ing toroids.

\section{${ }_{72} B$. The ATLAS Trigger and Data Acquisition systems}

73 In total the ATLAS detector has around $10^{8}$ electronic 74 channels, resulting in events with an average size of $1.7 \mathrm{MB}$. 
Table I: Table with LHC upgrade phases and nominal parameters [2][3].

\begin{tabular}{|c|c|c|c|c|c|c|c|c|}
\hline & \multicolumn{2}{|c|}{ Run 1} & \multirow{4}{*}{ 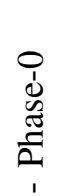 } & Run 2 & \multirow{3}{*}{ 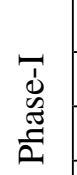 } & Run 3 & \multirow{4}{*}{ 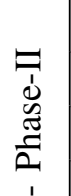 } & Run 4 \\
\hline & 2011 & 2012 & & $2015-17$ & & $2019-21$ & & $2023-$ \\
\hline Center of mass Energy $\sqrt{s}(\mathrm{TeV})$ & 7 & 8 & & $13-14$ & & 14 & & 14 \\
\hline Luminosity $\left(\mathrm{cm}^{-2} \mathrm{~s}^{-1}\right)$ & \multicolumn{2}{|c|}{$8 \times 10^{33}$} & & $1 \times 10^{34}$ & \multirow{4}{*}{ ஸै } & $2 \times 10^{34}$ & & $5 \times 10^{34}$ \\
\hline Bunch spacing (ns) & \multicolumn{2}{|c|}{50} & \multirow[t]{3}{*}{$\sqrt{3}$} & 25 & & 25 & \multirow{3}{*}{$\tilde{n}$} & 25 \\
\hline Number of interaction/event, $\langle\mu\rangle$ & 10 & 20 & & $\sim 27$ & & $\sim 55-80$ & & $\sim 140$ \\
\hline Total Integrated luminosity $\left(\mathrm{fb}^{-1}\right)$ & \multicolumn{2}{|c|}{25} & & $\sim 100$ & & $\sim 300$ & & $\sim 3000$ \\
\hline
\end{tabular}

${ }_{75}$ As they are read for every proton bunch crossing, every $25 \mathrm{~ns}$, 100 the HLT. The detector data of these events is then read from 76 the total data volume is closer to $\sim 64 \mathrm{~TB} / \mathrm{s}$, unfeasibly large 101 the front-end electronics and stored in buffers in the Readout 77 to be recorded or processed with full precision in real-time ${ }_{102}$ System (ROS), waiting for HLT requests and decisions.

78 with LHC. Furthermore, only a small fraction of these events ${ }_{103}$ The HLT is software based, implemented mainly in C/C++, 79 contain a significant physics interest. The selection of which 104 and runs on a CPU computing cluster, under a a component 80 events should be kept for later analysis is made by the ATLAS ${ }_{105}$ framework named Athena [8]. The system was designed for ${ }_{81}$ trigger system [5], which probes the event data against a menu 106 multi-process event processing, running one HLT Processing 82 of desirable physical characteristics, typically the presence of ${ }_{107}$ Unit (HLTPU) in each CPU core. HLT executes chains of 83 a certain physical object (e.g. a highly energetic electron), that 108 reconstruction (feature extraction) algorithms followed by ${ }_{84}$ leaves a distinctive pattern in the detector. This menu contains 109 hypothesis testing (hypothesis) algorithms. Chains are seeded 85 a few thousands of different possibilities. The event selection 110 by the L1 RoIs. Each algorithm in a chain runs over the output 86 is hampered by background patterns which mimic the desired ${ }_{111}$ of the previous one. If the same algorithm is scheduled for ${ }_{87}$ objects. Each event has to be processed in real-time and the ${ }_{12}$ execution in different chains over the same data, then the first 88 data volume reduced by a factor of $10^{4}$ [6], [7] for offline ${ }_{113}$ execution output is cached and used on the remaining chains. 89 storage.

${ }_{114}$ In this way repeated calculations are avoided. The hypothesis 115 algorithm's job is to compare the features produced against

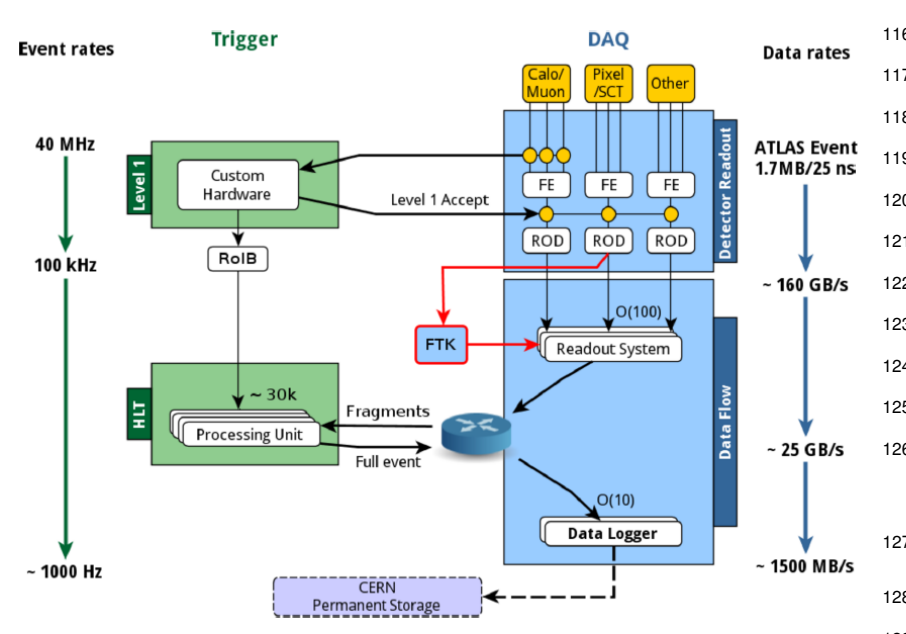
116 some configured hypothesis and accept or reject the events. ${ }_{117}$ The system was designed for early event rejection. It also 118 allows chains than run over partial data, requiring typically $2 \%$ ${ }_{119}$ to $6 \%$ of the full event data, to be processed in order to reject ${ }_{120}$ the events. For the rejected events the data is flushed from ROS ${ }_{121}$ system and the information of this collision is not retained. 122 This is the case for $99 \%$ of events. HLT has an average event ${ }_{123}$ processing time budget of $300 \mathrm{~ms}$. In this time it selects at 124 most one out of 100 events thus reducing the event rate for 125 permanent storage to about $1 \mathrm{kHz}$, translating to a data rate of 126 about $1.5 \mathrm{~GB} / \mathrm{s}$.

\section{TRIGGER ON GPUS}

128 ATLAS is investigating the execution of trigger recon129 struction algorithms on GPUs, as a potential solution to Figure 1: Schematic diagram of the ATLAS Trigger system ${ }_{130}$ meet Run 3 challenges of event reconstruction within the showing the input and output event rates and the expected ${ }_{131}$ trigger computing cluster constraints, mainly the available data rates at the different trigger levels.

${ }_{132}$ rack-size and the thermal extraction capability. As GPUs 133 are autonomous high performance computing devices, data ${ }_{90}$ The trigger system is divided in two levels, as shown in ${ }_{134}$ has to be transferred between the CPU host and the GPU ${ }_{91}$ Figure 1, the Level-1 (L1) and the High Level Trigger (HLT). ${ }_{135}$ device, imposing a data transfer overhead. The performance ${ }_{92} \mathrm{~L} 1$ is based on custom hardware and is located near the ${ }_{136}$ achieved, along with the code porting effort required, in terms ${ }_{93}$ detector. It uses a simple and fast reconstruction, over a coarse ${ }_{137}$ of manpower, will contribute to the architecture choices of ${ }_{94}$ granularity readout of the calorimeter and muon spectrometer, ${ }_{138}$ the trigger computing cluster upgrade. To aid the architectural ${ }_{95}$ to find Regions of Interest (RoI), where high transverse energy 139 choice a demonstrator implementation of several algorithms, ${ }_{96}\left(E_{\mathrm{T}}\right)$ objects like electrons $(e)$, photons $(\gamma)$, muons $(\mu)$ or ${ }_{140}$ most time consuming or demonstrating the worst luminosity ${ }_{97}$ jets are found. L1 takes a decision within a latency of $2.5 \mu \mathrm{s} 141$ scalability, was launched. It is supposed to conclude with the ${ }_{98}$ and selects at most one out of 400 events, thus reducing the ${ }_{142}$ indication of the cost/benefit estimates for various hardware ${ }_{99} 40 \mathrm{MHz}$ crossing rate to a maximum of $100 \mathrm{kHz}$ for input to ${ }_{143}$ compositions of the future HLT computing cluster. 


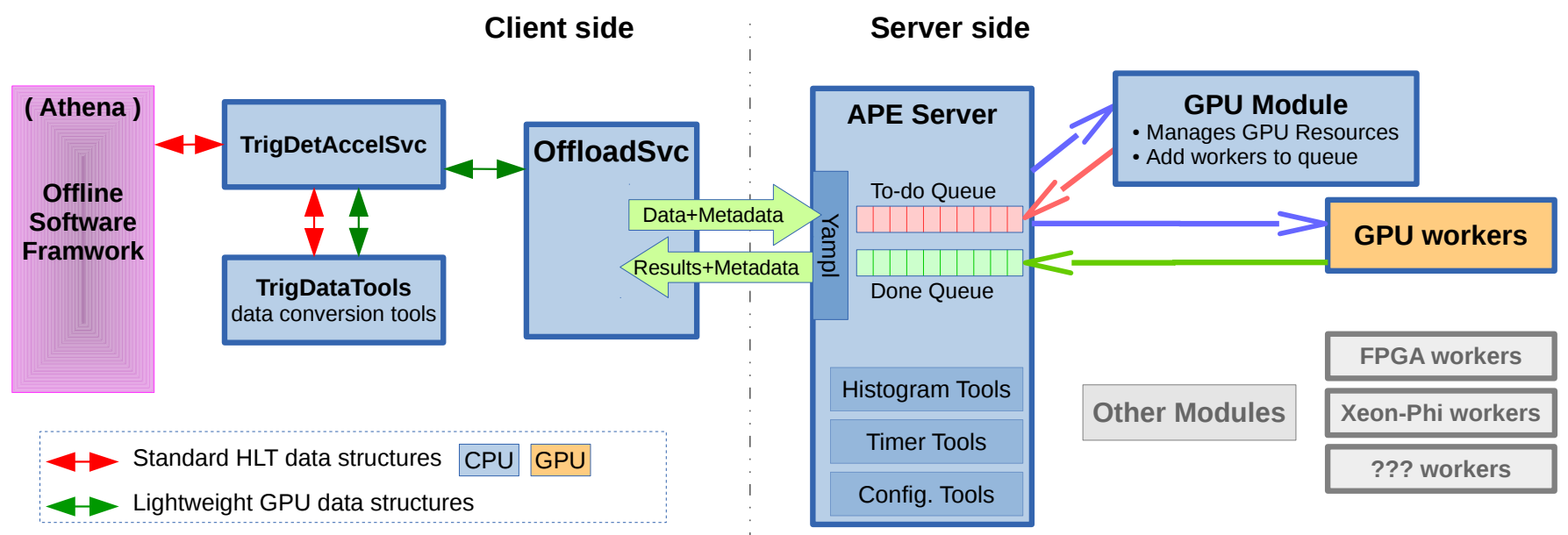

Figure 2: Trigger on GPU framework schematic. ATLAS framework (Athena) clients request offloading to the TrigDetAccelSvc. TrigDetAccelSvc uses TrigDataTools for data conversions between the client and server structure. Data and meta-data is then sent through the offloading service. The server reads the meta-data and hands the request to the proper module. The module appends the data to a unique data space and gives it to a new worker. The worker is appended to a to-do queue that is managed by the server. After execution, the worker goes to a done queue and the server hands the result back to the client.

144 The trigger GPU project demonstrator comprises ID, 178 The data to be processed are then sent to the GPU through ${ }_{145}$ calorimeter, muon and jet reconstruction algorithms. From an 179 the offloading service (OffloadSvc) to the APE server. After ${ }_{146}$ initial analysis, taking into account expected speedup due to ${ }_{180}$ processing, the offloading service sends the result back to the ${ }_{147}$ Amdahl law, the following set of algorithms was selected to ${ }_{181}$ detector specific acceleration service, which in turn sends it 148 be ported to a GPU architecture: 182 back to the HLT algorithm, after converting back to the Athena 149 - Inner Detector data preparation, seed making and track- 183 data structures. following algorithms.

- Calorimeter cell clustering algorithm.

- Muon tracking algorithms, based on Hough transform.

- Jet finding Anti- $k_{t}$ algorithm [9], [10].

Nvidia cards with the CUDA [11] framework were chosen

${ }_{155}$ for the demonstrator, based on the hardware quality, maturity 156 of the technology and framework, the good framework support 157 and lower porting effort.

\section{${ }_{158}$ A. GPU Acceleration framework}

159 The demonstrator implements a client-server architecture, 160 based on the Accelerator Process Extension (APE) frame${ }_{161}$ work [12], to offload and process HLT data, as shown in ${ }_{162}$ Figure 2. This allows a reduction of the resources needed 163 as the services of one server are available to many clients 164 as well as separation of concerns, where the APE server is 165 only responsible for computing on GPU while HLT only for 166 processing on CPU.

${ }_{167}$ 1) Client Side: The client side is implemented in the HLT. ${ }_{168}$ For the reconstruction of data from each ATLAS sub-detector 169 an algorithm requesting the GPU-accelatared processing is 170 developed. These algorithms extract the input data from the ${ }^{204}$ It is the module that is responsible for executing the work ${ }_{171}$ detector, request GPU-acceleration process of the data through ${ }^{205}$ items by placing them in to-do queue. It is also responsible 172 an acceleration service (TrigDetAccelSvc), and inject the result ${ }^{206}$ for sending the result of the computation back to the HLT. 173 back to the HLT.

${ }_{207}$ Modules and workers are specific of each detector.

174 The TrigDetAccelSvc uses TrigDataTools to convert back 208 By using the accelerator abstraction and the modular struc175 and forth the sophisticated Athena data structures to ones 209 ture, APE can exploit any kind of computing resource, such as 176 suitable for GPU implementation. Each sub-detector uses its 210 GPUs, FPGAs or Xeon-Phi, as long as modules and workers 177 own Acceleration service and data export tool.

211 are provided for such technologies. 
237 suppressing the noise contribution. The noise suppression is 213 The GPU demonstrator project started with an evaluation ${ }^{238}$ achieved by making the cell clustering dependent on the 214 of the HLT algorithms. It highlights the most interesting 239 neighbouring cells energy significance (S/N), the latter given 215 algorithms to port to GPUs by each sub-system: the inner ${ }^{240}$ by the ratio of the energy deposition with respect to the average ${ }_{216}$ detector tracking, the calorimeter cell clustering, the muon ${ }^{241}$ electronics and pile-up noise in that cell. However, this kind of 217 tracking and the jet finding algorithms.

242 clustering requires more computation than what is required by ${ }_{218}$ The ID tracking is the most time consuming part of the ${ }^{243}$ simpler algorithms. Thus, the Topological Cluster algorithm is ${ }_{219}$ system, followed by the calorimeter clustering, muon tracking 244 only used in the latest stage of the original ATLAS trigger and 220 and jet maker.

245 in the offline reprocessing of the accepted events.

246 The calorimeter cell clustering classifies the detector cells

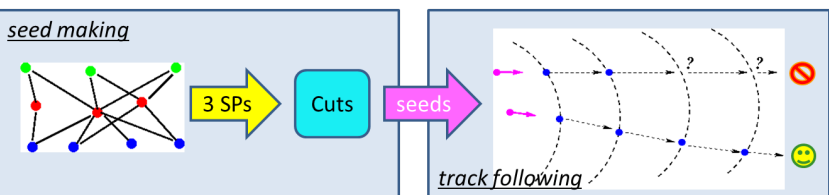

$\sum$ Spacepoints $>10^{5} \sum$ SP triplets $>10^{9} \sum$ Seeds $>10^{4} \sum$ Tracks $>10^{3}$ 247 in three groups, according to the cells signal-to-noise ratio. ${ }_{248}$ Cells with higher ratio, usually above four, are called SEED 249 cells. Beside those, cells are classified as GROWING, usually 250 if the energy is two times higher than the noise, or TERMI251 NAL, which are remaining cells with absolute energy above 252 zero. Each SEED cell starts a cluster formation, as shown 253 in Figure 4. The clusters grow by iteratively including the

Figure 3: Inner detector seed making and track-following 254 neighbours of SEED or GROWING cells. TERMINAL cells are algorithms schematics. Compatible clusters in inner layer are 255 added to clusters to form the outer layer. Two different clusters paired to form seeds. Seeds are then paired with outer layer 256 are merged if they share a SEED or GROWING cell.

clusters to form triplets. Triplets are then followed through the 257 The GPU implementation of this algorithm, the Topological full detector to form track candidates. A decision algorithm ${ }_{258}$ Automaton Clustering (TAC), is a parallel oriented implementhen selects the final tracks. 259 tation of the TC algorithm. It has to keep the TC properties 260 and produce the same results. The algorithm starts with the

221 1) Inner detector: The inner detector reconstruction starts 261 cells classification. At this stage, the work space is simplified 222 with the decoding of the raw data [13]. It then clusters neigh- 262 into pairs of cell and a neighbour. This abstraction assures an 223 bouring activated sensors (hits), using a cellular automaton 263 evenly distribution of workload across all GPU cores. Then 224 algorithm [14]. Compatible clusters in the two inner most 264 the SEED cells are ordered so that each cluster will have a 225 layers are paired to form objects known as seeds, left side 265 unique tag, the position of the SEED cell in the ordered list. 226 of Figure 3. Seeds are paired with the clusters in the outer 266 The clustering starts after that. The clustering is based on a ${ }_{227}$ layer to form triplets of space-points. Then track-following 267 cellular automaton algorithm. Each thread evaluates a pair of 228 algorithm starts and extrapolates the triples of space-points 268 cells and makes the cluster tag propagate according with the ${ }_{229}$ (SPs) through the full detector, to form track candidates, as 269 rules specified before. This process continues till the iteration ${ }_{230}$ shown in right side of Figure 3. After the track-following 270 cells do not change their tag. The set of cells in each cluster 231 a large number of tracks candidates is formed due to the 271 is the result shipped to the HLT client.

232 significant detector occupancy. Therefore, at the final stage, an 233 hypothesis algorithm selected best tracks from all candidates. 272

\section{RESULTS}

273 Preliminary results of the trigger GPU demonstrator are

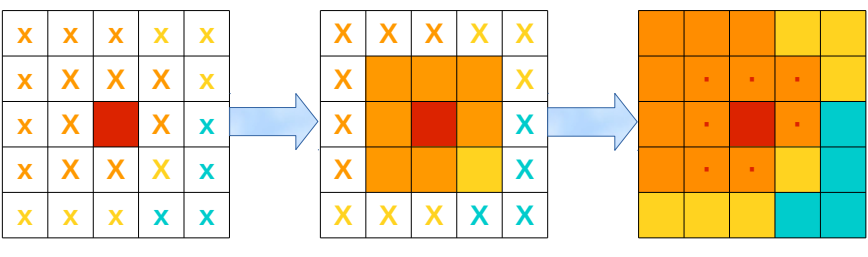
274 presented below. The gross figure of merit for the demonstrator 275 is the throughput expressed as the number of events processed 276 per second using the specific combination of hardware and 277 software. The benefits of faster execution on the PC with GPU 278 have to be compared against performance of same power or 279 same cost machine with only CPUs. Fair comparisons have to 280 assume comparable performance of the algorithms of which 281 an example is presented below. In addition to the throughput 282 the scalability of the implementations has to be assessed. This Figure 4: Calorimeter cell clustering algorithm schematic. The ${ }^{282}$ is achieved by measuring the algorithm processing time as a algorithm starts by classifying the cells in 3 groups according ${ }_{284}$ function of input data size. to $\mathrm{S} / \mathrm{N}$ value: SEEDS > GROWING > TERMINAL. SEED cells initiate clusters, with a defined unique cluster tag. SEEDS and GROWING cells tag is passed to their neighboring cells, if they 285 A. Inner detector are tagged with higher $\mathrm{S} / \mathrm{N}$ ratio. The algorithm stops when ${ }_{286}$ The per-event execution time of the track seeding algorithm, no cell tag is modified.

287 as a function of the number of space points, is shown in ${ }_{288}$ Figure 5. The plot compares the standard CPU serial imple234 2) Calorimeter: The ATLAS Topological Cluster (TC) [15] 289 mentation against the parallel version ported to GPU. This 235 algorithm joins the calorimeter detection units, known as cells, 290 test was performed on a machine with an Intel ${ }^{\mathrm{TM}} \mathrm{Xeon}^{\mathrm{E} 5}$ ${ }_{236}$ to form three-dimensional energy deposition clusters whilst $2912695 @ 2.3 \mathrm{GHz}$ and a Nvidia ${ }^{\mathrm{TM}}$ Tesla K80. The data set used 


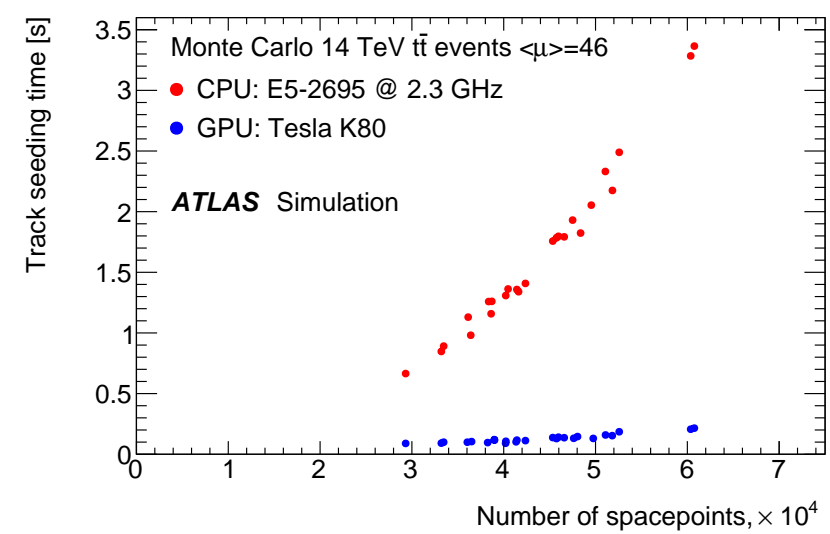

307 per bunch-crossing. The results presented are obtained after 308 the complete Trigger Clustering execution. The histogram 309 shows that both distributions are in very good agreement, with 310 the mean number of cluster agreeing within $0.1 \%$ for both ${ }_{311}$ implementations.

312

\section{CONCLUSIONS}

313 The LHC instantaneous luminosity for Run 3 will double 314 compared to Run 2. For the ATLAS trigger system, higher 315 luminosity will require more computation power to exploit 316 the full potential of the LHC. GPUs are massive parallel 317 architectures with high computing throughput and efficiency, 318 in terms of operations per watt, making them interesting

Figure 5: Timing of the Inner Detector (ID) track seeding ${ }_{319}$ solutions for the trigger computing cluster upgrade.

algorithm for the full detector. The red dots represent the ${ }_{320}$ A GPU trigger demonstrator prototype is being implestandard HLT ID algorithm, running on a single CPU core, ${ }_{321}$ mented to assess the potential of such a system. For it, a the blue dots show the algorithm ported to GPU. The timing ${ }_{322}$ server-client system was chosen to handle the trigger requests is shown as a function of input data size, a number of space $_{323}$ for GPU data processing. The demonstrator covers a set of points from which the seeds are formed [16].

324 algorithms from all sub-detectors. The ID tracking algorithm 325 has already demonstrated a very significant speed-up of 17

326 times. For the calorimeter cell clustering, the results showed ${ }_{292}$ consisted of Monte Carlo simulated $t \bar{t}$ events, for a scenario of ${ }_{327}$ an almost perfect agreement between the CPU and the GPU ${ }_{293} 14 \mathrm{TeV}$ collisions and a mean value of 46 proton interactions ${ }_{328}$ versions of the algorithm. Muon and jet algorithms are in the 294 per bunch crossing, representing a typical scenario for Run $3 ._{329}$ final implementation stage. The final stage of integration is 295 This plot shows that the GPU implementation of the $\mathrm{ID}_{330}$ ready and further tests are going to be performed to include 296 tracking algorithm is already up to 17 times faster than the ${ }_{331}$ detailed measurements of the throughput per unit cost for ${ }_{297} \mathrm{CPU}$ version and its performance scales linearly in the region ${ }_{332}$ various architectural choices.

298 of interest.

\section{B. Calorimeter cell clustering}

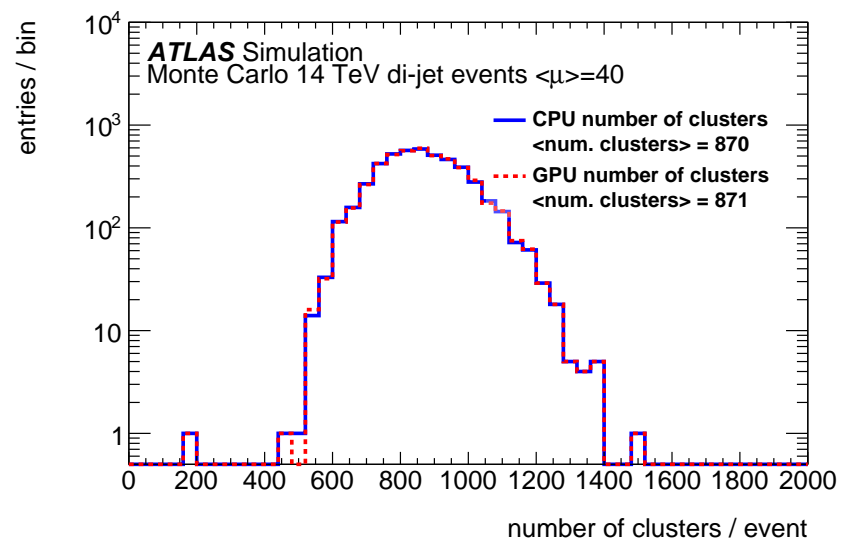

Figure 6: Number of calorimeter clusters reconstructed using ${ }_{355}^{354}$ the standard CPU cell clustering algorithms and the algorithm 356 ported to GPU. The blue line represents the CPU standard ${ }^{357}$ algorithm. The red dashed line represents the GPU cell clus- ${ }_{359}$ tering based on a Cellular Automaton algorithm [17].

The number of clusters reconstructed per-event is shown in ${ }_{301}$ Figure 6. The histogram compares the standard CPU serial ${ }^{364}$ 302 implementation against the ported GPU parallel version of the ${ }_{366}^{365}$ 303 algorithm, for a data sample of QCD di-jet events, simulated ${ }_{367}$ 304 using Monte Carlo simulated events for a scenario of $14 \mathrm{TeV}^{368}$ 305 collisions, with leading-jet transverse momentum above $20_{370}^{369}$ ${ }_{306} \mathrm{GeV}$ and a fixed number of 40 simultaneous interactions 371

$333 \quad$ REFERENCES

334 [1] L. Evans and P. Bryant, LHC Machine, JINST 3 (2008) S08001.

35 [2] ATLAS Collaboration, Letter of Intent for the Phase-I Upgrade of the ATLAS Experiment, Tech. Rep. CERN-LHCC-2011-012. LHCC-I-020, CERN, Geneva, Nov, 2011. https://cds.cern.ch/record/1402470.

[3] ATLAS Collaboration, Letter of Intent for the Phase-II Upgrade of the ATLAS Experiment, Tech. Rep. CERN-LHCC-2012-022. LHCC-I-023, CERN, Geneva, Dec, 2012. https://cds.cern.ch/record/1502664.

[4] ATLAS Collaboration, The ATLAS Experiment at the CERN Large Hadron Collider, JINST 3 (2008) S08003.

[5] ATLAS Collaboration, Performance of the ATLAS Trigger System in 2010, Eur. Phys. J. C 72 no. 1, (2012). http://dx.doi.org/10.1140/epjc/s10052-011-1849-1.

[6] ATLAS Collaboration, Technical Design Report for the Phase-I Upgrade of the ATLAS TDAQ System, Tech. Rep. CERN-LHCC-2013-018. ATLAS-TDR-023, CERN, Geneva, Sep, 2013.

[7] ATLAS Collaboration, Performance assumptions for an upgraded ATLAS detector at a High-Luminosity LHC, Tech. Rep. ATL-PHYS-PUB-2013-004, CERN, Geneva, Mar, 2013. http://cds.cern.ch/record/1527529.

[8] ATLAS Collaboration, ATLAS computing: Technical design report,

[9] M. Cacciari, G. P. Salam, and G. Soyez, The anti- $k \_t$ jet clustering algorithm, Journal of High Energy Physics 2008 no. 04, (2008) 063-063. http://dx.doi.org/10.1088/1126-6708/2008/04/063.

10] ATLAS Collaboration, Run II Jet Physics: Proceedings of the Run II QCD and Weak Boson Physics. 2007.

360 [11] J. Nickolls, I. Buck, M. Garland, and K. Skadron, Scalable parallel programming with CUDA, Queue 6 no. 2, (2008) 40. http://dx.doi.org/10.1145/1365490.1365500.

12] S. Kama, Triggering events with GPUs at ATLAS, Tech. Rep. ATL-DAQ-PROC-2015-013, CERN, Geneva, May, 2015. https://cds.cern.ch/record/2016471.

[13] ATLAS Collaboration, Algorithms for the ATLAS high-level trigger, 51 no. 3, (2004) 367-374. http://ieeexplore.ieee.org/stamp/stamp.jsp?arnumber=1310527.

[14] D. Emeliyanov and J. Howard, GPU-Based Tracking Algorithms for the ATLAS High-Level Trigger, Tech. Rep. ATL-DAQ-PROC-2012-006, CERN, Geneva, May, 2012. https://cds.cern.ch/record/1450130. 
372 [15] W. Lampl et al., Calorimeter Clustering Algorithms: Description and Performance, Tech. Rep. ATL-LARG-PUB-2008-002.

ATL-COM-LARG-2008-003, CERN, Geneva, Apr, 2008. https://cds.cern.ch/record/1099735.

376 [16] https://twiki.cern.ch/twiki/bin/view/AtlasPublic/

$377 \quad$ HLTTrackingPublicResults\#Phase_I_Upgrade_public_plots.

378 [17] https://twiki.cern.ch/twiki/bin/view/AtlasPublic/HLTCaloPublicResults\#

379 HLT_Calo_performance. 